\title{
VILLA Y COMARCA FUNCIONAL EN GALICIA
}

\author{
Román Rodríguez González
}

\section{RESUMEN}

Desde los años sesenta Galicia experimenta, como consecuencia de la difusión del proceso de urbanización y la introducción dentro de los circuitos económicos occidentales, una fuerte secuencia de transformación de las estructuras socioeconómicas y territoriales preexistentes. Estas dan como resultado la formación de un modelo de organización espacial dual entre un eje litoral densamente urbanizado, donde se concentra la población y la fuerza económica, y un interior de carácter rural compartimentado en comarcas.

El desarrollo urbano y funcional de las villas de las áreas rurales da como resultado la formación de áreas de influencia de carácter supramunicipal que se corresponden con comarcas funcionales. Estas se superponen a las tradicionales comarcas naturales como piezas básicas de la articulación de los espacios agrarios de Galicia.

Palabras clave: villa, comarca, función, espacio rural.

\section{RÉSUMÉ}

Dés les années soixante, La Galice subit une grande transformation dans les estructures socio économiques et territoriales préexistantns, comme conséquence de la diffusion du procés d'urbanisation et de son introduction dans les milieux économiques occidentaux. Ceci entraine comme résultat la formation d'un modéle d'organisation spatial duel, entre un axe littoral trés urbanisé oú sont concentrés la population et la force économique, et une zone intérieur á caracteristiques rurales divisée en régions.

Le développement urbain et fonctionnel des petites villes rurales aboitit á la formation de zones d'influence á caractére supramunicipal qui correspondent á des regions fonctionnells, qui se superposent aux traditionnelles régions naturelles comme piliers distributeurs des espaces agraires de La Galice.

Monts clé: petite ville, région, fonction, espace rural.

En el conjunto nacional se mantiene una percepción de Galicia como una Comunidad Autónoma de fuerte carácter rural. Evidentemente, esta apreciación inicial no está exenta de cierta lógica si tenemos en cuenta que en la misma, y a fecha de 1993, el 30,2\% de la población activa pertenece al sector primario (frente al 10,1\% del total del estado), y que la 
situación periférica, e incluso, la imagen que se plantea desde los medios de comunicación de masas, inciden en esta idea genérica de ruralidad.

Sin embargo, la realidad objetiva se muestra en la actualidad lejos de esta visión estereotipada. Las últimas décadas se asocian a un intenso proceso de transformación de las estructuras sociales, económicas y territoriales preexistentes, motivado por el arrastre que supondrá la inserción de España dentro de los círculos económicos occidentales de corte capitalista que favorecen la industrialización y el desarrollo urbano del territorio nacional (Souto González, 1988).

El proceso de urbanización afecta a la redistribución de los grupos humanos y actividades económicas sobre el espacio, y, también, a los sistemas de organización territorial. En este sentido, el territorio se entiende a partir de los centros urbanos y sus respectivas áreas funcionales, tanto en los entornos de las principales ciudades del sistema regional, como en las áreas rurales interiores, estructurándose estas a partir de las pequeñas ciudades, o villas cabeceras de comarca que en ellas se localizan.

En este último aspecto se centra la presente aportación. Se planteará como el crecimiento de estos núcleos, asociado a toda una serie de transformaciones globales en las estructuras preexistentes, favorece la creación de un sistema de articulación espacial organizado a partir de las áreas de influencia, o comarcas funcionales, de modestos centros urbanos que adquieren un carácter supramunicipal en función del alcance y atracción de sus actividades terciarias.

\section{Urbanización y nuevos modelos territoriales}

La estructura territorial gallega se entiende a partir del contraste existente entre el eje urbano-periurbano que desde la ciudad de El Ferrol llega a la frontera portuguesa siguiendo en trazado de la Autopista del Atlántico, y el espacio interior de la región que presenta un marcado carácter rural, donde los espacios urbanos se reducen a las capitales provinciales interiores y a las villas cabeceras. Partiendo de cualquier indicador o variable socioeconómica a utilizar (densidades demográficas, localización industrial, modos de poblamiento,...) el contraste es fácilmente apreciable hasta el punto de que existe un consenso en la apreciación de dos modelos diferenciados de organización espacial en Galicia (Torres Luna, Pazo Labrador y Santos Solla, 1991).

De forma muy sintética, el eje marcado por el subsistema urbano litoral se define como un espacio de elevadas densidades demográficas, con numerosos asentamientos de importancia donde se generalizan procesos de metropolitarización y suburbanización en torno a las ciudades de Vigo, Pontevedra, Santiago, A Coruña y Ferrol. Aquí, sobre una limitada franja espacial, se concentra gran parte de la carga empresarial y de la riqueza económica de Galicia. Por el contrario, las áreas no integradas en este corredor ofrecen una lectura diferenciada. Espacios en los que se desarrollan actividades agrarias con una organización espacial fijada a partir de la configuración de subsistemas comarcales centrados en un pequeño núcleo terciario, la villa cabecera, introduciéndose en la organización espacial de las ciudades mayores en una situación de dependencia para actividades de cierta especialización (Lois González, 1993).

En el eje urbano litoral se articula una red urbana policéntrica, bipolar y con una complementación funcional entre las ciudades y los núcleos urbanos de menor tamaño (Precedo Ledo, 1987). Esta se organiza en dos regiones urbanas. Una al norte, centrada en el área metropolitana de A Coruña, y que incluye la comarca de Ferrol y todo el entorno rururbano del Golfo Artabro. Y otra, al sur, a partir del complejo urbano que conforma Vigo, su área metropolitana, Pontevedra y el sector de las Rias Baixas. En medio de ambas, 
Santiago, muy reforzada en la última década al incrementar sus funciones tradicionales con la condición de capital político-administrativa de Galicia, vive un proceso de fuerte expansión urbana que irradia a sus municipios limítrofes, especialmente Teo, Ames y Brión, hasta el punto de afirmarse que se está configurando una nueva área metropolitana (Ferrás Sexto y Lois González, 1993). Fuera de este eje, las provincias de Lugo y Ourense se estructuran territorialmente a partir de las respectivas capitales provinciales, ciudades medias con una funcionalidad mayoritariamente terciaria, y de diversos subsistemas comarcales de asentamientos (Lois González y Rodríguez González, 1995).

En este contexto dual, la funcionalidad y el papel de las villas como elementos organizadores del espacio varía según su localización en el espacio. Los pequeños núcleos urbanos más próximos a las ciudades principales del eje litoral experimentan un creciente proceso de integración y complementación funcional, ofertando funciones específicas (localización industrial, ocio y turismo,...) en una situación de dependencia respecto a las ciudades mayores (Souto González, 1988). Las villas situadas en las áreas de fuertes densidades urbanas de las Rías Baixas, el Golfo Artabro y la Mariña Lucense, marcan un modelo de articulación policéntrico, con numerosos núcleos de tamaño demográfico e importancia funcional similar, donde es muy difícil, debido a las numerosas interrelaciones internas, establecer áreas de influencia individuales (Precedo Ledo, 1987). Por el contrario, los centros situados en los espacios menos urbanizados del interior, destacan por su capacidad de organizar subsistemas de asentamientos de base supramunicipal a partir de las funciones de servicios con las que cuentan (Rodríguez González, 1995).

Esta dialéctica establecida sobre el espacio gallego se crea a partir de un proceso histórico en el que intervienen factores varios como la distribución de los recursos potenciales de aprovechamiento humano, el modelo de organización socioeconómica o las diferentes políticas económicas regionales. Se parte de una situación inicial de desigual reparto de los recursos. Las franjas costeras de Galicia, con la excepción del litoral coruñés conocido como «A Costa da Morte», son áreas de una gran fertilidad agrícola que favorece la aparición de una agricultura intensiva y de un modelo de hábitat denso y disperso. A su vez, las características morfológicas del litoral son muy apropiadas para el desarrollo de funciones portuarias. Sobre estas se organizan dos actividades de gran trascendencia: el comercio marítimo y la pesca. Retrocediendo en el tiempo, la aparición de excedentes agrícolas comercializables en la Edad Media y Moderna, va ser un impulso importante para la fundación y creación de «villas» con una orientación hacia el comercio atlántico (Villares Paz, 1988). Del mismo modo, a mediados del siglo pasado estas actividades experimentan una serie de transformaciones que favorecen la aparición de una base empresarial alrededor de la pesca, centrada en la construcción naval y en la manipulación de las capturas. Ambas circunstancia impulsan históricamente el incremento de los umbrales de ocupación humana y la diversidad funcional de las áreas marítimas (Souto González, 1990). Recientemente el auge del turismo estival, centrado en las playas, es un recurso que también favorece la acumulación demográfica y económica en las áreas costeras.

Sobre esta situación de reparto diferencial de los recursos potenciales, la situación de Galicia como área periférica tanto de los ejes de desarrollo europeos como nacionales, es un factor más que incide en el agravamiento de los contrastes territoriales (Pérez Vilariño, 1990). La situación de Galicia en la periferia del sistema productivo europeo inserta a la comunidad gallega dentro del clásico modelo centro-periferia de J. Friedman, por el que las innovaciones y cambios surgidos en los espacios centrales se concentran primero en determinados puntos de los periféricos. En estos se produce un proceso acumulativo de crecimiento y transformación de sus estructuras productivas internas, económicas y sociales, con lo que se agrava las desigualdades interterritoriales de base (Precedo Ledo y 
Rodríguez Martínez-Conde, 1991). Por esto, después de la integración de Galicia dentro del sistema económico de corte capitalista que se generaliza en España en la segunda mitad del S. XX, el impacto del nuevo modelo de funcionamiento económico tenderá a la potenciación de economías de concentración en áreas espaciales restringidas.

Esta dualidad territorial se refuerza, así mismo, por la política económica regional de los anos sesenta y setenta. La concentración de inversión estatal en materia de infraestructuras, junto a la localización de empresas públicas y grandes consorcios empresariales atraídos por los atractivos generados por la estrategia de Polos de Desarrollo, en los espacios que hoy forman el corredor urbano-litoral (Vigo y A Coruña en el I Plan de Desarrollo y Vilagarcía en el III), acentúa la tendencia concentradora de actividades y población en estas áreas.

Por su parte, en el interior, se produce un paralelo proceso de ajuste de unas estructuras agrarias precapitalistas y autárquicas a un nuevo sistema de producción orientado hacia el mercado. En estas comarcas se lleva a cabo una fuerte modernización y transformación de las explotaciones agropecuarias (nuevas estrategias productivas, mecanización, introducción de insumos,...). Este proceso supone la ruptura del precario equilibrio existente hasta este momento entre la población y los recursos campesinos. Se produce un excedente de mano de obra campesina que da como resultado un trasvase de recursos humanos desde los espacios agrarios interiores de Galicia en dirección a áreas de crecimiento industrial, tanto gallegas como nacionales y europeas. Tal situación supone para los territorios rurales una constante pérdida de fuerza demográfica en contraste con los espacios litorales que se ven favorecidos por ser zonas de recepción de trabajadores procedentes de las comarcas interiores.

Desde comienzos de siglo, en razón del ya aludido reparto diferencial de recursos, las áreas más pobladas de Galicia se fijan en los entornos costeros. A su lado, un interior eminentemente rural, mantenía una situación demográfica estable posibilitada por un ajuste espontáneo entre el elevado crecimiento natural de la población y las pérdidas de efectivos vía migración americana, o hechos puntuales de sobremortalidad (Hernández Borge, 1992). El medio rural, durante la primera mitad del S. XX. mantenía unas estructuras familiares caracterizadas por un elevado número de miembros. Situación necesaria dentro de la lógica productiva de un modelo de aprovechamiento agrícola de autoconsumo y subsistencia. Tal circunstancia permite a las zonas agrarias mantener unos umbrales poblacionales de importante peso relativo en los totales de Galicia. Será con el comienzo de un conjunto de transformaciones favorecidas por la incidencia del proceso urbanizador, cuando se produzca, a partir de los sesenta, un doble fenómeno caracterizado por un reforzamiento demográfico de los sectores urbanos-litorales y un fuerte retroceso de población en los municipios rurales

Esta tendencia es explicable, en primer lugar, a partir de la oferta creciente de empleo no agrario en las villas y ciudades en crecimiento del litoral. Y, en segundo lugar, por el masivo éxodo de población desde los espacios agrarios. Éxodo que implica una perdida directa de potencial demográfico y la aparición de drásticas consecuencias en la evolución natural de la población. Para ilustrar tal afirmación se recurre a la observación de la dinámica demográfica de los municipios gallegos entre 1960 y 1991. En este período se suceden todo un conjunto de modificaciones cuya plasmación demográfica a nivel territorial supone la individualización de dos espacios con dinámicas evolutivas dispares. En primer lugar, los municipios que incrementan sus efectivos poblacionales tienden a localizarse en dos áreas definidas: el entorno de las Rías Baixas prolongándose por el Val del Ulla hasta Santiago, y el Golfo Artabro, alrededor de las ciudades de A Coruña y Ferrol. Son estos espacios que ganan población a un gran ritmo, íncrementando, algunos de ellos, 


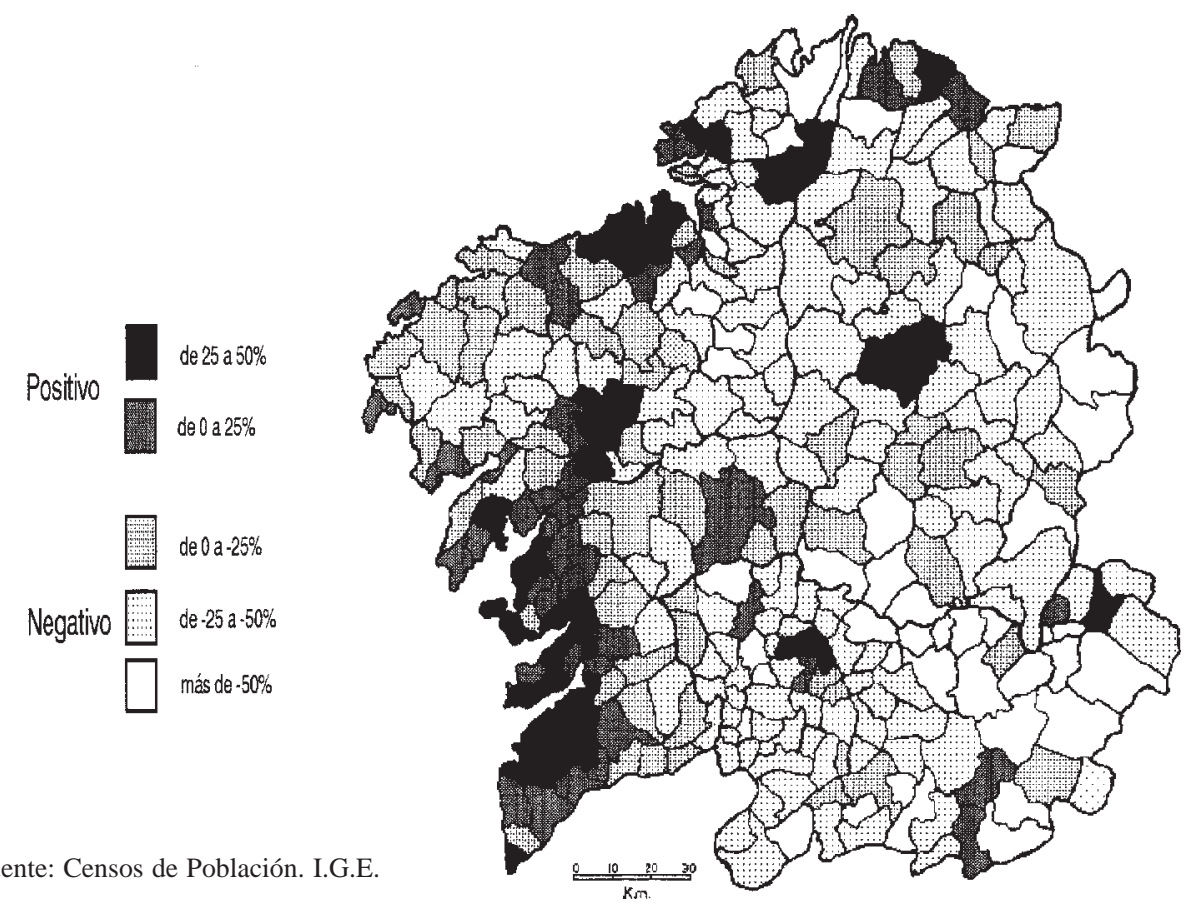

Evolución demográfica de los municipios gallegos entre 1960 y 1991.

sobre todo en las áreas metropolitanas de Vigo y A Coruña, su población en más de un $25 \%$. Hacia el interior esta secuencia solo se presenta en las capitales provinciales y en aquellos municipios que cuentan con una cabecera comarcal de gran vitalidad con un crecimiento que permite, en este periodo temporal, compensar la pérdida de fuerza demográfica de su territorio rural. Aunque en ocasiones, el aumento de población del núcleo urbano no es capaz de compensar el masivo éxodo de los habitantes del medio rural (sobre todo en los municipio de gran superficie), con lo que el municipio, en conxunto, pierde población pese el incremento demográfico de la villa (Rodríguez González, 1995).

En frente a estos, la mayor parte de las entidades gallegas pierden población en las últimas tres décadas. En algún caso, especialmente en las áreas de montaña, el declive es tal que supone pérdidas de más de un 50\% de la población entre las fechas de referencia. Predomina, no obstante, una disminución para los espacios agrarios entre un 25 y un $50 \%$ del volumen inicial. Circunstancia que los relaciona, directamente, con la desigual incidencia que las improntas del proceso urbanizador tienen sobre los espacios en los que se desarrolla. Y, también, con la diferente capacidad competitiva que cada área presenta para adaptarse al nuevo modelo de organización socioeconómica y territorial marcado por el proceso urbanizador posterior a los sesenta.

\section{Incremento funcional de las villas y formación de áreas dependientes}

Frente a las áreas litorales de fuerte impacto del proceso urbanizador, el interior mantiene una estructura del territorio organizada a partir de las villas cabeceras. Alrededor de estas, se genera una serie de subsistemas de asentamientos humanos que dependen 
funcionalmente del núcleo central, correspondientes al área de influencia de sus actividades económicas. Junto a esta división primaria del espacio interior, organizativamente, se complementa la red urbana de la Galicia interior con las capitales provinciales respectivas (en algunos casos con otras ciudades medias), donde se localizan determinadas funciones de ámbito provincial o supracomarcal: sedes provinciales de diversos servicios, administración provincial,... (Lois González y Rodríguez González, 1995). Mientras, las villas rectoras de cada comarca, se sitúan en un escalón funcional que cubre gran parte de las demandas de la población de su entorno que, salvo para determinadas funciones muy específicas, no necesita desplazarse a las ciudades mayores del sistema urbano.

Presentando la organización espacial del interior gallego a partir de los subsistemas de asentamientos, definidos por las diferentes áreas de influencia de cada cabecera, es imprescindible el planteamiento de la fuerte vinculación establecida entre el núcleo central de la comarca y el territorio que organiza. Se tiene puesto de manifiesto la correlación existente entre la funcionalidad y la talla urbana de cada villa, con las características socioeconómicas de los entornos que organiza (Rodríguez González, 1995). Como se verá a continuación, para los espacios de montaña se individualiza una tipología de villa que difiere, en cuanto a sus variables geoeconómicas más destacadas, de las pequeñas ciudades rectoras de los espacios agrarios. Cada asentamiento presenta una adaptabilidad a las potencialidades con las que cuenta el territorio que organiza, y solo para casos concretos donde se desarrollaron fenómenos puntuales, como procesos de industrialización (As Pontes, Sarria,...) o la potenciación de una actividad concreta (caso de Monforte con el ferrocarril), esta relación no es directa. Se parte así, de una identificación de los diferentes espacios comarcales con sus villas. Villas que deben su centralidad dentro de los subsistemas de asentamientos a la posesión de actividades de servicios con capacidad para organizar el territorio en áreas de dependencia funcional.

El modelo de organización espacial del interior gallego detectado en la actualidad, tiene un origen en el tiempo coetáneo a la generalización de las transformaciones introducidas por el proceso de urbanización en Galicia. Se viene afirmando, en diferentes aproximaciones que se tienen llevado a cabo sobre la problemática conceptual de la comarca, que un término tan genérico y común como este es motivo de atención por numerosos investigadores y disciplinas que plantean muy variadas perspectivas de estudio. La inexistencia de una acepción única y universalmente válida de lo que se entiende por comarca, impide el establecimiento de una delimitación con un trazado estable de las mismas. En general, es posible definir lo que es una comarca a partir de la idea de «región» defendida por la escuela regionalista francesa, en el sentido de una área espacial individualizada por unos determinados caracteres del medio físico, con unas relaciones humanas internas que la dotan de singularidad y la diferencian de las restantes. Se presenta así a las comarcas como una «miniregión», en el sentido amplio del término.

Sobre este acuerdo inicial, se llevan a cabo diversas propuestas de comarcalización en base a diferentes criterios como pueden ser los rasgos naturales, los aspectos económicos, el sentimiento de pertenencia de la población,... Desde nuestra perspectiva de análisis se busca destacar el papel que las villas tienen en la configuración de espacios de gravitación en base al alcance de las actividades no agrarias que se localizan en ellas. Se entienden así las comarcas, para el espacio interior de Galicia, como territorios organizados a partir de un pequeño núcleo urbano desde el que se ejercen aquellas funciones orientadas al abastecimiento de los diferentes asentamientos humanos que quedan dentro de su área de influencia.

Uno de los principales caballos de batalla a la hora de la aceptación de cualquiera propuesta comarcalizadora, es el establecimiento de los límites que marcan y definen dicha 
unidad territorial. Tarea esta consumidora de grandes esfuerzos y muy difícil de precisar. Se defiende la existencia de tantas delimitaciones comarcales como criterios utilizados, e incluso, se tiene afirmado en algunos estudios pioneros en el ámbito de la comarcalización, que es un poco ingenuo trazar límites precisos a las comarcas, pues no los tienen (Casas Torres, 1973).

Al no tener hasta el momento un reconocimiento jurídico administrativo estable, la fijación de cualquiera división comarcal aparece cuestionada por dos motivos fundamentales. En primer lugar, las comarcas se comportan como un subsistema de estructuración territorial, vivo y mutante en el tiempo donde los diferentes agentes interaccionan con el espacio. La modificación de los sistemas de transporte, la transformación de las estructuras productivas, la introducción de nuevas actividades económicas, los cambios en los valores sociales y de comportamiento, la difusión de la información y otros factores diversos, hacen de las comarcas porciones del territorio que se adaptan a los diferentes procesos que se producen sobre ellas. Así mismo, y dentro de nuestra defensa de las comarcas como espacios de gravitación directa de las villas, los diferentes análisis preocupados por el establecimiento de las áreas de atracción de los servicios de los núcleos centrales, resaltan la existencia de unas áreas de indiferencia, franjas territoriales más o menos amplias, que pueden depender, indistintamente, de una u otra cabecera, cuestionándose cualquiera parcelación del territorio en base a las áreas de influencia de cada población central (Precedo Ledo, 1987).

Y, en segundo lugar, es necesario tener en cuenta que todo ejercicio de fijación de unos límites comarcales, responde a unos intereses particulares. Todo investigador persigue unos objetivos individuales y utiliza unos criterios y un método determinado, con lo que, en lógica, los resultados difieren entre ellos, siendo todos, si tal labor se realiza con rigor, igualmente válidos.

Junto a estos dos factores que cuestionan cualquier establecimiento de una delimitación rígida de los espacios comarcales, los sucesivos paradigmas desarrollados en la historia geográfica favorecieron una conceptualización del hecho comarcal diferenciada.

En respuesta a los enfoques deterministas introducidos por el positivismo en la ciencia geográfica, se recoge un primer concepto de comarca natural, como aquel espacio uniforme desde el punto de vista de los caracteres del medio físico. Las comarcas se delimitarían, así, a partir de una serie de elementos geomorfológicos destacados (una depresión, una alineación montañosa, un valle fluvial,...) que dota al paisaje de uniformidad. Posteriormente, con la intención de conjugar sobre el territorio las interrelaciones entre los elementos físicos y los humanos, y al amparo metodológico de la escuela regionalista francesa, se plantea un modelo de compartimentación comarcal en base a los elementos visibles del paisaje. Se refleja, con una visión histórica, como el hombre se adapta y transforma el territorio, dotando a las diferentes partes de este de una uniformidad paisajística en las que se basan dichas divisiones. Ya en la última mitad de siglo, y como consecuencia del creciente interés que en las investigaciones geográficas suscitaron los análisis sobre los sistemas de redes urbanas, aparece el concepto de comarca funcional, que, como se viene afirmando, se trata de un subsistema de asentamientos que depende funcionalmente de un núcleo central en el que se localizan actividades comerciales y de servicios. En las últimas aportaciones se detecta una doble tendencia al planteamiento de las comarcas como ámbitos territoriales de planificación e intervención socioeconómica, y se profundiza, bajo una vertiente humanista, en el sentimiento de pertenencia e identificación personal del hombre con el espacio en el que habita.

Profundizando en la comarca como un espacio de dependencia funcional, su configuración como territorios afectados por diferentes flujos socioeconómicos «a partir de» $\mathrm{y}$ «cara 
a» un determinado centro poblacional que dibuja una área de influencia en base a sus funciones (Vilá Valentí, 1994), es en Galicia reciente. Se marca esta a partir del proceso de urbanización y desarrollo terciario que afecta, a partir de los sesenta, a los tradicionales centros de mercado agrario, y que supondrá su crecimiento físico, demográfico y económico-funcional. La centralidad de los núcleos rectores de comarca se define a partir tanto de las actividades comerciales, como de los diferentes servicios, públicos y privados, que en ellos se localizan. Los servicios públicos permiten a las villas contar con unas áreas de influencia nítidas sobre el territorio, constituyéndose las comarcas como ámbitos espaciales para la satisfacción de una demanda colectiva. A nivel comercial es más problemático el establecimiento de una delimitación estable, ya que las zonas de atracción marcadas por el comercio son fluctuantes, dependiendo de múltiples factores (nivel de renta, deseo personal, medio de transporte,...). Pero es evidente que en cada espacio comarcal existe una villa que por la oferta comercial que presenta, es capaz de cubrir las necesidades principales de la población de su entorno que, salvo para demandas muy específicas, no tiene necesidad de desplazarse a una entidad de mayor talla funcional.

\section{Comarca y villa, piezas maestras de la organización espacial}

Dentro de la clásica definición de comarca funcional se entiende aquel espacio concreto poblado y explotado por un grupo humano que aparece repartido en múltiples asentamientos, estando organizado por un núcleo cabecera que sirve a toda la comarca por medio de sus actividades terciarias (Casas Torres, 1973). Aquí late una premisa básica que afecta directamente a las villas del interior de Galicia: la importancia que estas juegan como centros abastecedores de bienes y servicios a la población de su entorno, o área de influencia funcional.

La atracción que ejerce cualquiera entidad urbana se basa en la capacidad de sus actividades de atraer a los habitantes de su radio inmediato que se desplazan a ella para consumir, o participar, en sus funciones. La noción de centralidad se vuelve básica para comprender el proceso de estructuración comarcal. El sentido de comarca, como área de influencia de un núcleo, se desarrolla, en primer lugar, en base a la potenciación comercial de las villas en las pasadas décadas, y, en segundo, gracias a las políticas de descentralización de servicios públicos que tienden a localizar determinados equipamientos (sanitarios, educativos, asistenciales,...) en las cabeceras para, desde ahí, atender con ese servicio concreto a una porción del territorio. Tal circunstancia propiciada por el proceso de urbanización, origina que la concepción de comarca natural planteada por Dantín Cereceda desde principios de siglo (espacio homogéneo desde el punto de vista de los componentes visibles del paisaje), pierda vigencia en favor de las comarcas funcionales, medidas a partir de las áreas de influencia de los núcleos urbanos, que pueden, o no, coincidir con los límites de una comarca natural (Bielza de Ory, 1993). De este modo aparece una trama superpuesta entre aquellos espacios que por su uniformidad paisajística se vienen considerando como comarcas, y aquellos otros que dependen funcionalmente de un núcleo urbano central. Se puede ejemplificar dicha afirmación presentado un caso concreto: la Comarca del Deza. Es esta una de las más claras de Galicia, situada en el noroeste de Pontevedra está formada por los municipios de Agolada, Dozón, Lalín, Rodeiro, Silleda y Vila de Cruces. Este espacio se dividía según varios autores (Otero Pedrayo, 1926; Fraguas Fraguas, 1958) en varias comarcas naturales: Deza, Camba y Trasdeza. Debiéndose la consideración como comarca única a la gravitación funcional que este espacio presenta respecto al núcleo urbano de Lalín, que articula y centraliza la vida económica, cultural y social de todo el entorno comarcal (Rodríguez González, 1995). 
La asociación para el espacio interior de Galicia entre proceso de urbanización y estructuración comarcal en base a comarcas funcionales, se entiende a partir de las dos premisas presentadas anteriormente (el desarrollo comercial y la localización de diversos servicios públicos) que potencian el desarrollo de estas poblaciones a partir de los anos sesenta. Con anterioridad a esta fecha, la centralidad de las villas tiene un antecedente, a nivel de atracción económica, en la influencia que ejercían como centros de intercambios periódicos en el medio agrario. Y a nivel administrativo por la localización en las cabeceras de comarca de las sedes de los Partidos Judiciales, primera división comarcal del territorio de especial trascendencia para el reforzamiento de la importancia de los centros beneficiados por la localización de tal servicio (González Mariñas, 1994).

Si antes de la generalización del proceso de urbanización, en Galicia se venía hablando de comarca en base a criterios histórico-culturales, y en función de afinidades paisajísticas y físico-ambientales, con las transformaciones que el mismo proceso introduce en los modos de organización socioeconómica y espacial, se pasa a hablar de comarcas como áreas de dependencia y/o influencia de un núcleo urbano central. En la atracción ejercida por estas cabeceras pesan de manera decisiva factores como la accesibilidad de cada núcleo, el nivel de servicios que suministra, su papel como centro comercial para la economía de la zona, y su oferta de empleo industrial y actividades de ocio.

Tras este planteamiento queda patente que en la concepción de comarca que se defiende aparecen tres elementos fundamentales: un territorio, un efectivo poblacional y un núcleo de población que desempeña el papel de centro comarcal (Vilá Valentí, 1994) Resulta evidente la asociación existente entre las villas y las comarcas, de modo que, para el interior de Galicia, hablar de subsistemas de asentamientos comarcales es sinónimo de hablar de subsistemas de villas.

Cada núcleo basa su entidad de organizador de la vida comarcal en los siguientes puntos. En primer lugar la concentración de actividades terciarias (justicia, enseñanza, sanidad, ocio, banca, comercio,...), favorece la aparición de un área de gravitación directa para el consumo de dichos servicios. En segundo término, se producen múltiples desplazamientos internos de población desde el espacio rural para acceder al núcleo para consumir las funciones que este oferta, o para trabajar, si es el caso, en los empleos no agrarios que el núcleo oferta. Y, en tercer lugar, la villa cabecera sirve como puerta de contacto de la población de todo el ámbito comarcal con el resto de los elementos del sistema urbano regional (vías de comunicación, noticias, trámites,...).

La diversidad espacial y los contrastes existentes en la realidad geoeconómica del territorio gallego, dan lugar a una heterogeneidad, tanto de las comarcas, como de los centros comarcales. La trama de villas cabeceras no es homogénea para el interior de Galicia, pues no todos los núcleos desempeñan las mismas funciones, ni tienen la misma relevancia. El proceso de urbanización, que sí afecta a todos ellos, se plasma en diferentes modelos de desarrollo que afectan a variables como el tamaño demográfico, la capacidad de atracción, la oferta funcional o el ritmo de consolidación física de los núcleos comarcales.

Entre los factores que más inciden en el desarrollo urbano de las villas interiores se encuentran, aquellos relacionados con las características socioeconómicas del propio territorio que organizan, ya que de estas va depender el umbral de demanda y consumo de las actividades terciarias que se pueden localizar en el núcleo central. También, el proceso de urbanización de los nodos rectores de comarca, puede ver condicionado su nivel de consolidación funcional por la competencia, tanto de las ciudades principales del sistema regional, como de otros núcleos de cierta entidad que pueden existir dentro del área comarcal. Por otra parte, la existencia, o no, de una estructura industrial constituye un 
factor básico para la comprensión de los procesos de crecimiento que afectan a determinadas cabeceras.

Partiendo de estas premisas se individualizan 31 asentamientos de población que consideramos villas cabeceras de comarca. En este grupo se manifiesta una gran diversidad en cuanto a indicadores básicos como el número de habitantes, el ritmo de consolidación edificativa, o la oferta económica que presentan. Sin embargo, el tratamiento en conjunto se basa, por una parte, en su acreditación como centros terciarios rectores de subsistemas de asentamientos comarcales, y, por otra parte, por ser núcleos afectados por una serie de transformaciones inducidas por la generalización del proceso de urbanización a partir de los años sesenta. Esto da lugar a un importante crecimiento demográfico, funcional y espacial de los núcleos cabecera de los espacios no costeros de Galicia.

\section{Realidad plural de las villas comarcales}

A continuación, y partiendo de una íntima relación entre las villas y el territorio que organizan, se presenta un modelo tipológico que recoge y sintetiza los diferentes procesos que actuaron sobre el desarrollo de los centros comarcales.

Una de las preocupaciones más importantes que se observa en numerosas investigaciones geográficas es el establecimiento de diversas tipologías que sinteticen los diferentes sucesos espaciales. Tal circunstancia, derivada del impacto que en las ciencias sociales supone el paradigma positivista, incide en la necesidad de modelizar los diferentes procesos que afectan al territorio. Pese a este interés, es frecuente la aparición de dificultades de índole epistemológico o conceptual para el establecimiento de cualquiera clasificación de los asentamientos urbanos. Gran parte de estos ejercicios se basan en técnicas estadísticas de análisis factorial multivariado donde se conjugan, fundamentalmente, numerosos índices geoeconómicos y sociodemográficos (Abellán García, Moreno Jiménez y Vinuesa Angulo, 1978).

Pese a la intención de los diferentes investigadores en obtener resultados objetivos y de validez o aplicación universal, toda definición, aunque formalmente parezca lo contrario, es de algún modo arbitraria o, en el mejor de los casos, conlleva una inevitable dosis de subjetividad (Precedo Ledo, 1988). Pese a esta salvedad de partida, es indudable la necesidad de establecer algún tipo de medición o baremo que nos permita encuadrar en grupos afines los diferentes procesos de crecimiento y desarrollo que inciden sobre las villas interiores de Galicia.

Sobre los núcleos urbanos gallegos se tienen hecho diversas aproximaciones a la variedad tipológica existente. Las más habituales son aquellas basadas en índices demográficos. Circunstancia lógica si tenemos en cuenta, en primer lugar, la accesibilidad y facilidad de manejo de las variables poblacionales y, en segundo, el carácter sintético y definitorio de estos índices (Abellán García, Moreno Jiménez y Vinuesa Angulo, 1978). Otro indicador también muy utilizado en el establecimiento de tipologías, es la actividad económica principal que marca la razón de ser de algunas villas y ciudades. Así se habla de villas portuarias, mineras, o de otro tipo.

La función que desempeñan los asentamientos urbanos en el espacio, con la introducción de la noción de centralidad, dio lugar a clasificaciones jerárquicas de las villas y ciudades en función de su capacidad de atracción sobre el entorno, realizadas, parte de estas, a partir de la aportación pionera de Miralbés Bedera y out., Galicia en su realidad geográfica, (1984).

Se llevan hecho, además, clasificaciones en base a criterios morfológicos: forma del plano, usos del suelo urbano, modelos arquitectónicos y de construcción espacial,.... y, 
también, se introducen variables de carácter perceptivo, basándose en la conciencia colectiva como medio para identificar lo que es o lo que no es núcleo urbano, y su importancia relativa dentro del sistema gallego de asentarnientos (Fumega Piñeiro, 1988).

Partiendo de estas premisas, interesa lograr una visión de síntesis que englobe los diferentes procesos de urbanización que afectaron desde 1960 al conjunto de pequeñas ciudades del espacio interior gallego. En este sentido, se tiene como soporte fundamental la caracterización del territorio articulado por cada núcleo urbano. Se plantea una relación directa entre las peculiaridades geoeconómicas de cada comarca y el tipo de villa que articula el respectivo subsistema comarcal de asentamientos. Pese a esto, en esta relación directa vila-comarca, pueden aparecer elementos que correspondan en exclusiva a los núcleos urbanos, provocando una disimetría entre el grado de desarrollo de la villa con respecto a su entorno (industrialización).

Se plantea una clasificación de los núcleos rectores de comarca del interior galego, que, partiendo de su inserción dentro del proceso de urbanización general de Galicia, incida en sus relaciones con el entorno territorial que organizan. En este punto se argumenta la existencia de tres tipos fundamentales de villas comarcales: villas de los espacios de montaña, villas de los espacios agrarios y villas de diversificación económica.

Esta triple división se presenta como marco básico de referencia para el estudio de los diferentes procesos de expansión que afectaron a cada cabecera en particular. El planteamiento integral de investigación que se presenta para cada una se fundamenta en el análisis de los diferentes agentes y elementos que intervienen y/o conducen la evolución económica, funcional, demográfica y física de estos núcleos.

Se pasa a continuación a exponer lo que se entiende por cada tipo de villa, haciendo hincapié, tanto en la necesaria visión de síntesis de los diferentes indicadores manejados como en los vínculos existentes entre estas y los subsistemas comarcales que organizan.

Como su propio nombre indica, las villas de los espacios de montaña son aquellas entidades de caracteres urbanos que concentran actividades terciarias, fundamentalmente comercio minorista y servicios públicos, para abastecer a los habitantes de las áreas montañosas interiores. Estas se corresponden con una amplia parcela territorial que se extiende por el sector oriental de la Comunidad Autónoma. Aquí se localizan una serie de pequeños núcleos cuyo umbral demográfico es, a fecha de 1991, siempre inferior a 2.000 habitantes, pero que se consideran auténticos centros terciarios rectores de los espacios comarcales de montaña (Torres Luna, Lois González y Pérez Alberti, 1993). Tal circunstancia, derivada de su funcionalidad, las dota de un papel singular dentro de la organización territorial de estas áreas, siendo el primer escalón dentro de la jerarquía del sistema urbano, posibilitando su consideración como verdaderas cabeceras de comarca (Casas Torres, 1973).

A nivel demográfico, aparte del volumen similar, coinciden estas villas en un ligero reforzamiento en las últimas décadas, con un incremento de población oscilante entorno a un 25\% entre 1960 y 1991. Incremento que contrasta con una acusada desolación que afecta a su espacio rural que en el mismo periodo pierde entorno al $50 \%$ de sus habitantes (el vaciamiento humano de las comarcas montañosas es un proceso general que afecta a la totalidad del Estado Español).

Esta circunstancia se debe a que las áreas de montaña salen muy perjudicadas del conjunto de transformaciones que, desde los anos sesenta, inciden en la modificación de los modelos tradicionales de organización económica, social y territorial que predominaban hasta este momento (Lois González, 1993). Estas transformaciones se pueden sintetizar, por una parte, en un cambio de las estructuras productivas agrarias, de un modelo de aprovechamiento autárquico y autoconsumista (donde era necesaria gran cantidad de mano 
TIPO DE VILLAS

de economía diversificada

de espacios agrarios

de espacios de montaña

TAMAÑO DE LOS NÚCLEOS DE POBLACIÓN

más de 10.000 habitantes

de 5.000 a 10.000

de 2,000 a 5.000

menos de 2.000

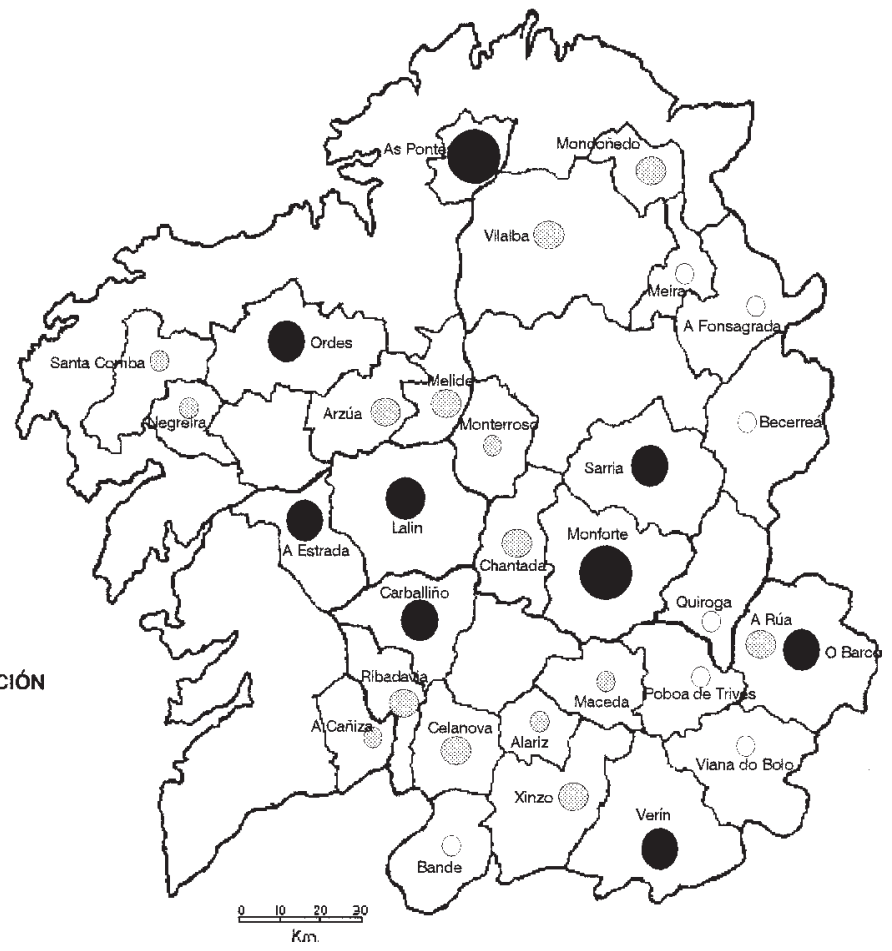

Tamaño y tipos de villas del interior de Galicia.

de obra para mantener la actividad), a otro en el que la producción final agrícola se orienta a los mercados urbanos, y donde el excedente poblacional encuentra una salida en la emigración. Y, por otra parte, en una progresiva pérdida de capacidad competitiva por el aislamiento respecto a las áreas más dinámicas de la región y los condicionantes del medio físico (clima, altitud, pendientes,...). Esto da como resultado que en las últimas décadas se produzca una desolación masiva de estos entornos, un abandono de las tierras de cultivo y una creciente dependencia económica de los habitantes de la montaña, vía subsidios, de las diferentes administraciones.

En este contexto de declive demográfico y económico estas cabeceras son los únicos lugares donde se aprecia, en las últimas décadas, un mínimo crecimiento demográfico, constructivo y económico/funcional.

Se estructuran así las comarcas montañosas de Galicia como subsistemas de asentamientos que se corresponden con las áreas de influencias de las pequeñas villas de montaña. Núcleos que marcan un radio de atracción medido por el alcance de los servicios que se localizan en ellas, y que se caracterizan por la presencia de una oferta que cubre las necesidades primarias de su población.

Partiendo de la definición primogénita de un núcleo urbano, como aquel centro abastecedor de bienes y servicios a la población, se comprende la razón de ser de los asentamientos considerados, villas de los espacios agrarios. Estas poblaciones son las herederas de los centros periódicos de intercambios agrarios en el medio rural, las ferias, siendo tratados, de forma tradicional, como los puntos centralizadores de la vida económica y social de las parroquias y aldeas de su entorno (Miralbés Bedera y Casas Torres, 1974). 
La definición como villa de espacio agrario implica una clasificación y tratamiento íntimamente relacionado con la actividad económica primaria y con el papel que desempeña en el territorio: centros de ámbito supramunicipal con una funcionalidad orientada al suministro de las demandas de los habitantes de su comarca para cuestiones, tanto relacionadas con el consumo directo, como con la propia actividad agropecuaria.

El análisis del proceso de urbanización de estas debe relacionarse con las profundas transformaciones que afectaron a la estructura productiva del campo desde los anos sesenta. Con anterioridad a su generalización, la presencia de modestos centros en los que se localizaban actividades artesanales y terciarias, venía dado tanto por la originalidad del poblamiento gallego, muy disperso en un gran número de entidades diminutas, como por la disposición de las líneas del relieve que dan lugar a la compartimentación del espacio en pequeñas comarcas naturales. Así mismo, la comprensión del sistema de asentamientos urbanos, evoluciona en el tiempo a partir de múltiples impulsos y decisiones individuales (Precedo Ledo, 1988). Teniendo, en este sentido, vital trascendencia para la existencia de algunas villas factores de índole histórico como la localización en los caminos de pelegrinación a Santiago (Melide, Arzúa,...), la existencia de enclaves fácilmente defendibles (Vilalba, Ribadavia,...), o la vinculación con el poder religioso (Celanova, Mondoñedo,...).

De una situación de estancamiento durante la primera mitad de siglo, estas cabeceras experimentan una inflexión, a partir de los sesenta, saldada con un crecimiento sin precedentes de sus índices demográficos, económicos y funcionales. Esta secuencia de cambios en los tradicionales mercados agrarios, se produce en función de las transformaciones de la estructura productiva agropecuaria. Como se destaca en múltiples estudios, en las últimas décadas se pasa de un modelo económico agrario autoconsumista, con unos mínimos excedentes comercializables, a otro donde su producción se especializa y se introduce en los circuitos de mercado, originando una transformación total de las relaciones económicas y sociales de los habitantes del medio rural. La modernización de las explotaciones agrícolas trae consigo, aparte de una reducción de la mano de obra empleada en el campo, una necesidad de consumo de diversos «inputs», imprescindibles dentro de la nueva estrategia de producción. La oferta de maquinaria, fertilizantes, piensos, servicios técnicos,... y otras demandas del sistema productivo agrícola, van ser funciones que comienzan a desarrollarse y centralizarse en los núcleos comarcales de los espacios agrarios.

El número de habitantes de las villas de los espacios agrarios oscila entre los $2.000 \mathrm{y}$ los 5.000 habitantes. Este volumen demográfico está en relación directa con variables como el grado de desarrollo agrícola-ganadero de cada comarca (marca el umbral de demanda de su área de dependencia), la existencia de agroindustrias en el núcleo (favorece la aparición de empleos complementarios), o la proximidad respecto a las ciudades del sistema urbano regional (por la competencia que supone a nivel comercial y de servicios). Así, frente a cabeceras centralizadoras de espacios comarcales de fuerte carga y productividad agropecuaria, donde existe un mínimo aparataje agroindustrial, y donde se ejerce una atracción monocéntrica se cuenta con otro subgrupo donde la actividad primaria se encuentra en declive y se produce una competencia funcional por parte de núcleos de mayor rango. Para las primeras (Xinzo, A Rúa, Vilalba, Melide y Ribadavia) se manejan umbrales poblacionales superiores a los 3.000 hbt., con unos ritmos de crecimiento entre 1960 y 1991 entorno al 225\%. Mientras, las segundas (Celanova. Mondoñedo, Arzúa, Alariz, A Cañiza, Santa Comba y Negreira), presentan un volumen y dinamismo inferior.

Junto a los anteriores, el sistema de villas del espacio interior de Galicia se completa con una serie de pequeñas ciudades que coinciden en la presentación de una base económica en la que se combinan actividades terciarias (comercio y servicios), con otras de carácter productivo e industrial. En la observación de las variables geográficas que definen a este 
grupo de entidades urbanas sorprende el espectacular ritmo de crecimiento demográfico, edificativo y funcional, que les afecta a partir de la década de los sesenta. En esta franja temporal el conjunto de transformaciones que incidieron en las relaciones sociales y económicas, impacta de manera decisiva en estos puntos, favoreciendo un rápido proceso de urbanización y desarrollo.

En primer lugar, estos núcleos gozan de una localización estratégica dentro de su ámbito comarcal: puntos de cruce de los ejes viarios intracomarcales. Esto favorece la atracción sobre la población de los espacios próximos, siendo, a la vez, el punto de mayor accesibilidad respecto a la red viaria regional. Tal circunstancia les supone una serie de ventajas locacionales que se manifestarán como básicas para garantizar la centralidad respecto a cada subsistema. Y, en segundo lugar, y como en el caso de las villas de los espacios agrarios, las transformaciones que inciden en la modernización del sector agropecuario en las últimas décadas, favorecen un incremento de su funcionalidad.

La circunstancia que individualiza a estas pequeñas ciudades es el interesante proceso de industrialización endógena, a partir de la potenciación de un sistema local de pequeñas y medianas empresas. Como se defiende en diversos estudios (Souto González, 1988), la orientación que, a partir de estas fechas, sigue el modelo económico nacional cara a un sistema de economía capitalista y de mercado, favorece el desarrollo industrial y urbano de Galicia. Obviando iniciativas individuales y esporádicas, de escaso éxito por las dificultades del contexto económico y político, las villas interiores de Galicia eran vacíos industriales con anterioridad a estas fechas. A partir de aquí, y gracias a una serie de ventajas que ofertaban este tipo de entidades dentro del nuevo entorno socioeconómico (locacionales, recursos financieros, disponibilidad de mano de obra flexible,...) se experimentará un momento de transformación de primitivas actividades artesanales en PYMES dinámicas.

Esta fase evolutiva se detecta en cabeceras como Ordes, Sarria, Lalín, Verín, A Estrada y O Barco, mientras que otras villas consideradas de economía diversificada, presentan impulsos de crecimiento más individuales y específicos. As Pontes basa su proceso de urbanización de los últimos anos en el arrastre que supone la ubicación del gran complejo industrial de Endesa. Monforte de Lemos es una pequeña ciudad, de gran tradición histórica, que vinculó gran parte de su desarrollo actual a la presencia y potenciación del ferrocarril. Y O Carballiño presenta un proceso de urbanización que debe entenderse a partir de la fortísima inyección de capital procedente de la emigración americana, y a la gran importancia de sus balnearios.

Así mismo, estas villas se convierten en puntos de localización de diversos servicios públicos y privados, y de un importante número de establecimientos comerciales. Esta oferta garantiza un considerable grado de atracción funcional sobre la población de su entorno, lo que contribuye a reforzar su proceso de crecimiento.

Tales circunstancias, dan como resultado que los núcleos anteriores experimenten un rápido crecimiento poblacional. Las villas ofertan un mercado laboral de actividades terciarias y secundarias que les permite alcanzar, a lo largo de las tres últimas décadas, unos índices de crecimiento medio entorno al 350\%, y unos volúmenes demográficos siempre superiores a los 5.000 habitantes. Así mismo, el proceso de urbanización que afecta a estas entidades contribuye a que, poco a poco, ganen una imagen de pequeña ciudad que servirá de reclamo para el retorno de numerosos emigrantes. La trascendencia del capital humano y financiero procedente de la emigración es una pieza importante dentro de las secuencias de crecimiento de gran parte de las villas gallegas. Su aporte se deja sentir, tanto en un aumento del número de residentes de los núcleos urbanos, ya que en su retorno eligen como lugar de residencia las ciudades o villas en lugar que sus parroquias 
de origen, como en la aparición de nuevas actividades económicas promovidas por los retornados.

A modo de conclusión, el proceso de crecimiento de las villas cabeceras en las últimas décadas se introduce dentro de la secuencia de cambio global que afecta, a partir de los años sesenta, a las estructuras socioeconómicas y a los modelos de organización espacial del conjunto nacional. Funcionalmente estas pequeñas ciudades incrementan sus niveles funcionales y refuerzan su capacidad de atracción sobre el espacio circundante, circunstancia esta que da lugar a la formación de áreas de influencia de las actividades centrales de las villas. Estas dan como resultado la formación de un entramado de comarcas funcionales entendidas como subsistemas de asentamientos de ámbito supramunicipal, en los que se compartimenta el territorio agrario del interior de Galicia. Pues las áreas litorales, que experimentan un intenso dinamismo urbano, plantean la formación de espacios de características metropolitanas y rururbanas.

Las villas se convirtieron, así, en puntos de concentración económica y humana con una principal misión: servir de Lugar Central del espacio rural que la rodea. Por esto, a medio plazo, su futuro se encuentra en una encrucijada debido a que una serie de fenómenos que actualmente se están desarrollando (reducción de las distancias respecto a las ciudades principales, crisis demográfica del espacio rural, frenos a la producción agropecuaria...) amenazan la continuidad de este modelo organizativo y pueden sumir a las, hasta el momento dinámicas cabeceras, en una situación de estancamiento. Situación que no debe tener, en principio, ni una lectura positiva ni negativa, sino que debe entenderse como una consecuencia lógica de la constantemente mutante organización de la vida en sociedad.

\section{Bibliografía}

ABELLÁN GARCíA, A. y aut.: Propuesta de tipología para ciudades españolas de tipo medio. Revista de Estudios Geográficos. Madrid, 1978.

BIELZA DE ORY, V.: Reflexiones sobre la comarcalización en España. En Vázquez Barquero y Precedo Ledo, coordinadores, Desarrollo Local y Comarcalización. Consellería de Presidencia da Xunta de Galicia, 1993.

CASAS TORRES, J.M.: La selección de núcleos de población cabezas de comarca para el bienio 1972-73. Revista Geographica, n 2. Zaragoza, 1973.

FERRÁS SEXTO, C. y LOIS GONZÁLEZ, R.C.: Estructura urbana de las áreas metropolitanas gallegas. La estructura urbana de Santiago. ¿Un área metropolitana en proceso de formación? En Papeles de Geografía, nº 19. Secretariado de Publicaciones de la Universidad de Murcia. 1993.

FUMEGA PIÑEIRO, F.X.: La percepción del sistema gallego de asentamiento. En Villares Paz R., coordinador: La ciudad y el mundo urbano en la historia de Galicia. Ed Tórculo. Santiago de Compostela, 1988.

GONZÁLEZ MARIÑAS, P.: Territorio e identidades. Galicia como espacio administrativo. EGAP, Xunta de Galicia. Santiago de Compostela, 1994.

HERNÁNDEZ BORGUE, J.: Sobre o estado da poboación galega. En Actas ó Congreso Internacional da Cultura Galega. Consellería de Cultura da Xunta de Galicia. Santiago de Compostela, 1992.

LOIS GONZÁLEZ, R.C. y RODRÍGUEZ GONZÁLEZ, R.: O retroceso da población campesiña e a crise da vida parroquial en Galicia. Revista de Investigación Pontenova, $\mathrm{n}^{\circ} 0$. Deputación de Pontevedra. 1995.

LOIS GONZÁLEZ, R.C.: Los cambios en la ocupación del espacio en la montaña gallega. En Pérez Alberti, A. y out.: La evolución del paisaje en las montañas del entorno de los caminos jacobeos. 
Consellería de Relacións Institucionais e Portavoz do Goberno da Xunta de Galicia. Santiago, 1993.

LÓPEZ GONZÁLEZ, A. y RODRÍGUEZ GONZÁLEZ, R.: La comarca como ámbito de estudio en la Geografía de Galicia. Actas al XIII Congreso Nacional de Geografía. Sevilla, 1993.

MIRALBÉS BEDERA, R. y out.: Galicia en su realidad geográfica. Fundación Barrié de La Maza. A Coruña, 1984.

MIRALBÉS BEDERA, R. y CASAS TORRES, J.M.: Los mercados periódicos en Galicia. Instituto de Geografía Aplicada del C.S.I.C. Madrid, 1974.

PÉREZ VILARIÑO, J.: As cidades alineadas do Atlántico e a economía política metropolitana en Galicia. En Revista Galega de Economía, n ${ }^{\circ}$. Consellería de Economía da Xunta de Galicia. Santiago, 1990.

PRECEDO LEDO, A. y RODRÍGUEZ MARTÍNEZ-CONDE, R.: El modelo de transición urbana en un área periférica. En Géographie Sociale, nº 10. Université du Caen. Le Mans, 1989.

PRECEDO LEDO, A.: Ciudad y desarrollo regional. Síntesis, Madrid, 1996.

PRECEDO LEDO, A.: Galicia: estructura del territorio y organización comarcal. COTOP, Xunta de Galicia. Santiago de Compostela, 1987.

PRECEDO LEDO, A.: La red urbana. Síntesis, Madrid, 1988.

RODRÍGUEZ GONZÁLEZ, R.: Lalín. Un proceso de urbanización e desenvolvemento. Deputación de Pontevedra, 1995.

SOUTO GONZÁLEZ, X.M.: Vigo. 100 anos de historia urbana. Ed Galaxia. Vigo, 1990.

SOUTO GONZÁLEZ, X.M.: Xeografia Humana. Ed Galaxia. Vigo, 1988.

TORRES LUNA, Ma. P. de, LOIS GONZÁLEZ, R.C. y PÉREZ ALBERTI, A.: A montaña galega. Home e espacio. Servicio de Publicacións da Universidade de Santiago de Compostela. 1993.

TORRES LUNA, Ma.P. de, PAZO LABRADOR, A.J. y SANTOS SOLLA, X.M.: Galicia, rexións de constrastes xeográficos. Servicio de Publicaciones de la Universidad de Santiago. 1991.

VILÁ VALENTÍ, J.: El papel de los geógrafos en la comarcalización. Ponencia al XIII Congreso Nacional de Geografía. Sevilla, 1994.

VILLARES PAZ, R.: A Historia. Ed Galaxia. Vigo, 1988.

VINUESA ANGULO, J. y VIDAL DOMÍNGUEZ, Ma J.: Los procesos de urbanización. Síntesis, 1991. 